\title{
Stimulus delay and the reduction of errors in the transfer of stimulus control*
}

\author{
CHARLES H. BROWN and MARK E. RILLING \\ Michigan State University, East Lansing, Michigan 48824
}

\begin{abstract}
Pigeons initially trained on a simultaneous discrimination of line orientation (S1) were subsequently transferred to a wavelength discrimination (S2). Three transfer procedures were employed. The abrupt-transfer Ss were "abruptly" switched from S1 to the S2 dimension. The stimulus-compounding Ss were trained on a compound stimulus consisting of S1 and S2 displayed in superimposition prior to the presentation of S2 alone. The stimulus-delay Ss were trained on a compound stimulus in which the presentation of the $\mathbf{S} 1$ component was delayed for successively longer intervals as a result of a correct response to the preceding trial. Stimulus-delay Ss transferred by responding to S2 prior to the presentation of $\mathrm{S} 1$ and the resulting formation of the compound. Ss transferred by the stimulus-compounding and the abrupt-transfer procedures displayed 5 and 10 times as many errors to the S2 dimension, respectively, as Ss receiving the stimulus-delay procedure.
\end{abstract}

An $\mathrm{S}$ forms a discrimination by learning to emit responses in the presence of one stimulus and by withholding responses in the presence of a second stimulus. Terrace (1963b) has shown that Ss may transfer from one discrimination to another without making errors. The absence of errors resulted from training pigeons to a compound stimulus consisting of an established wavelength discrimination displayed in superimposition with the to-be-transferred-to linetilt discrimination and gradually "fading out" the wavelength cue by reducing the intensity of that dimension.

Reynolds (1961) has demonstrated that either dimension of a compound-stimulus display may come to exert stimulus control. Thus, when more than one stimulus dimension is correlated with reinforcement, it is unclear which element of the stimulus display is, at any point in time, exerting stimulus control. Terrace's (1963b) transfer procedure employs a compound stimulus in which the relative intensity of the stimulus elements is systematically varied over several sessions. Considerable economy in the transfer program could be realized if a mechanism were available which would indicate that point in the transfer program by which the second dimension in the stimulus display has acquired stimulus control.

Several procedures have been generated which minimize that occurrence of errors in the transfer of stimulus control from one dimension to another. These

*This paper represents part of a thesis submitted by the senior author to the Department of Psychology, Michigan State University, in partial fulfilment of the requirements for the degree of Master of Arts. This research was supported, in part, by Grant 2RO1 MH 18342 from the National Institute of Mental Health, awarded to Mark E. Rilling. The authors wish to thank M. Ray Denny and Robert L. Raisler for their insightful appraisal of an earlier version of the manuscript. $R$ eprints $m$ ay be obtained from Charles H. Brown, Department of Psychology, Michigan State University, East Lansing, Michigan 48824. procedures have been developed for normal and retardate humans (Moore \& Goldiamond, 1964; Touchette, 1968), as well as for a variety of animal Ss (Ray, 1967; Schusterman, 1966; Westbrook \& Miles, 1970) and the training sequence in each of these procedures has required the $S$ to respond to a compound stimulus. These transfer programs, along with Terrace's (1963b), share the disadvantage that the point in the training sequence by which the to-be-transferred-to stimulus dimension has acquired stimulus control remains procedurally elusive.

This problem has been addressed by Touchette (1971). Working with retarded boys as Ss, Touchette produced an elegant procedure permitting the identification of the trial upon which the to-be-transferred-to dimension exerted stimulus control. Ss are first trained to criterion on a simultaneous discrimination. In the next session, the dimension of transfer, S2, is presented in conjunction with the dimension exerting control, S1. Correct responses to the compound are reinforced, terminate the trial, and affect the subsequent trial by progressively delaying the onset of the S1 component of the compound, thus having the consequence of presenting the S2 dimension alone for successively longer durations. Similarly, errors terminate the trial and reduce the interval between trial onset and the presentation of $\mathrm{S} 1$ to form the compound on the subsequent trial.

Behavior is under control of the original dimension as long as the $\mathrm{S}$ waits for the presentation of the compound before responding. Stimulus control is transferred to the second dimension when the $S$ responds before the compound is presented. The efficacy of Touchette's procedure is revealed by his $S$ 's errorless performance.

However, the status of Touchette's procedure may be questioned for several reasons. First, Touchette's Ss had extensive experimental histories. The extent to which errorless performance was a function of the S's history is 
unclear. Second, and of greater interest, while Touchette's stimulus-delay technique does reveal when the dimension of transfer acquires control, it is unclear whether this technique functions merely as a measuring device or if it also facilitates acquisition of the stimulus control it is measuring.

The present research addressed the former issue by adapting the delay technique to experimentally naive pigeons. In so doing, we hoped to be able to extend the application of Touchette's procedure to infrahuman organisms. In approaching the second issue, we observed that Touchette's procedure was unique in permitting the onset of the two stimulus dimensions which constituted the compound to occur asynchronously. It was the progressive departure from the simultaneous presentation of the stimulus components that enabled Touchette to detect when the second dimension was capable of maintaining stimulus control. Specifically, the strategy of the present work was to juxtapose three transfer procedures for the purpose of comparing posttransfer performance on the second stimulus dimension. One group of $\mathrm{Ss}$ was transferred via Touchette's stimulus-delay procedure. The posttransfer performance of these Ss was compared with the performance of stimulus-compounding Ss, which received equivalent training to the compound-stimulus display, but the stimulus elements constituting this display were always presented synchronously. The juxtaposition of these two groups of Ss should reveal whether the progressive asynchrony of the stimulus elements in Touchette's stimulus-delay procedure functions as a passive measuring device or if it actively facilitates the transfer of stimulus control.

The posttransfer performance of these two groups was compared with the performance of abrupt-transfer Ss which received the second stimulus dimension without the benefit of training on a compound display. This comparison should reveal the extent to which training on a compound display, via either the stimulus-delay or the stimulus-compounding procedure, facilitates performance on the second stimulus dimension.

\section{METHOD}

\section{Subjects}

Twenty-four locally obtained white Carneaux pigeons served as Ss. The birds were experimentally naive and were maintained at $80 \%$ ad lib weight throughout the experiment.

\footnotetext{
Apparatus

A standard Lehigh Valley 3-key experimental pigeon chamber was used. Inline digital display stimulus projectors transilluminated the center and left response keys with horizontal and vertical lines or red and green lights. The centers of the response keys were $8.25 \mathrm{~cm}$ apart and $25 \mathrm{~cm}$ from the floor of the chamber. The right response key was not used. The projected lines were white bars $5 \mathrm{~mm}$ in width and $25 \mathrm{~mm}$ in length, bisecting the response key. The lines could be projected either on a dark key or on a red or green background. The brightness of the hues was adjusted to appear equal to the $\mathrm{E}$.
}

Conventional electromechanical programming apparatus was used to control the experiment and was housed in an adjoining room. A punched paper-tape reader was used for program control, and the serial order of responses and latencies were recorded on a printout counter.

\section{Procedure}

Training on the S1 Stimulus Dimension. Ss were randomly assigned to one of the three groups: (1) stimulus-delay, (2) stimulus-compounding, and (3) abrupt-transfer. Each group was comprised of eight birds. All Ss received 20 sessions of simultaneous discrimination training on the $\mathrm{S} 1$ dimension. Vertical and horizontal lines served as $\mathbf{S}+$ and $\mathrm{S}-$, respectively. Stoddard (1968) and Touchette (1969) have suggested that line orientation may not be a unidimensional variable and may present more than one basis for discrimination. In the present study, in which the transfer of stimulus control was restricted to 50 trials, line orientation was not used as the dimension of transfer in order to minimize the expression of individual differences.

A trial began with the presentation of a stimulus on each of the two keys and was terminated when a peck occurred to one of the stimuli. No maximum limit was imposed on the duration of a trial. Correct responses were reinforced by producing 3-sec access to mixed grain. Responses to the incorrect stimulus were not reinforced and they entered the $S$ into a 5 -sec intertrial interval. Each session was terminated following the delivery of 50 reinforcements. An intertrial interval of $5 \mathrm{sec}$ preceded each trial; responses during this interval prevented the onset of the subsequent trial for an additional $5 \mathrm{sec}$. The experimental chamber was dark during the intertrial interval while, during the trial, both response keys and the houselight were illuminated. The onset of the houselight preceded the illumination of the keylights by $340 \mathrm{msec}$. The discriminative stimuli were presented on the response keys according to a modified Gellerman (1933) series. Following an error, the trial was repeated. This prevented a change in the stimulus configuration on the keys until a correct response occurred.

The Presentation of the S2 Stimulus Dimension. After the completion of 20 sessions of training on the S1 dimension, the Ss in each of the three groups were presented with a simultaneous wavelength discrimination in which a red light and a green light served as the $S+$ and $S-$, respectively. The transfer session consisted of 60 trials. The first 10 trials for all three groups were on the original line-tilt dimension and provided a warm-up series of trials. The subsequent 50 trials presented the S2 dimension in a manner specific to each group.

The stimulus-delay group was presented the $\mathbf{S} 2$ dimension, following Touchette's procedure. On the 11 th trial of the transfer session, the S1 dimension was superimposed on the S2 dimension in order to form a compound-stimulus display. A peck to the correct key increased the interval from trial onset to the presentation of the $S 1$ dimension on the next trial by $340 \mathrm{msec}$. For example, if no errors had yet occurred, the S2 dimension would be presented on the fourth transfer trial for $1.02 \mathrm{sec}$ prior to the superimposition of the $\mathrm{S} 1$ dimension to form the compound. Similarly, errors decreased by $340 \mathrm{msec}$, the interval from trial onset to the formation of the compound. Thus, the interval in which the $S$ waited for the presentation of the compound was titrated by the sequence of errors and correct responses emitted by the $S$. In this procedure, transfer was defined by that trial subsequent to the S's last response to the compound. When transfer occurred, the latency from the trial onset to the response was less than the scheduled duration from trial onset to the formation of the compound; thus, the compound was not presented.

The stimulus-compounding group was presented with the transfer session in the following manner. On Trials 11-39, a conventional compound-stimulus display was presented. On 


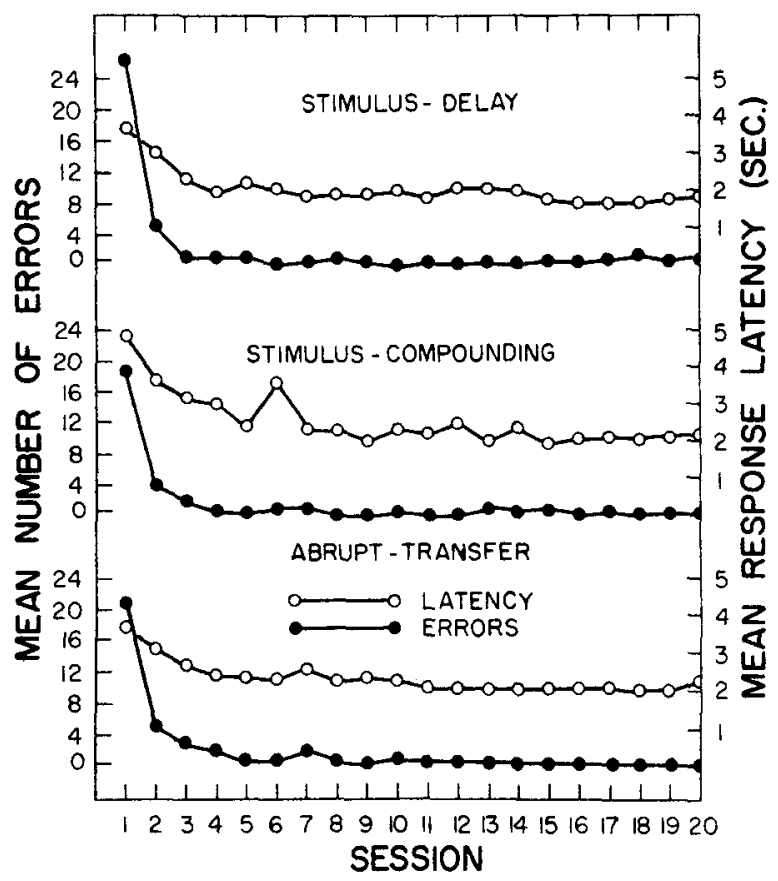

Fig. 1. The acquisition of the $S 1$ discrimination. The mean number of errors and response latency for $S$ s in each group on the 20 sessions of training on the $S 1$ discrimination.

Trials $40-60$, the $\mathrm{S} 2$ stimulus dimension was displayed alone. The 29 trials during which the compound stimulus was presented were determined by the fact that this was the maximum number of trials required for transfer by any $S$ in the stimulus-delay group. Thus, the Ss in the stimulus-compounding group received the compound display at least as many times as the Ss in the stimulus-delay group; the central difference between the two treatments was that the stimulus for the stimulus-compounding group was not modified by the progressive-delay feature.

The abrupt-transfer group received the transfer session as follows: S1, the line-tilt dimension, was presented for 10 trials; S2, the wavelength dimension, was presented for the subsequent 50 trials. This group was not presented with a superimposed or compound-stimulus display.

Training on the S2 Stimulus Dimension. On the session following the transfer session, the Ss in all three groups were presented the discrimination, following the identical oontingencies that the $S$ s received during training on the $S 1$ discrimination.

In the 20 training sessions preceding the transfer session, in the transfer session, and in the session following transfer, the performance of all Ss was assessed by two dependent variables: latency of response and percentage of errors. Latency was recorded up to 100 multiples of a $340-\mathrm{msec}$ time constant. Thus, the maximum latency included in the ensuing analysis was $34 \mathrm{sec}$. A correction procedure was in effect throughout the transfer session for the Ss in all three groups.

\section{RESULTS}

\section{Performance on the S1 Dimension}

Figure 1 presents the course of acquisition on the S1 discrimination for the Ss in all three groups. The left ordinate scales the mean number of errors for Ss in each group. The right ordinate scales the mean response latency for Ss in each group. As can be seen in Fig. 1, the distribution of errors and latencies follows the same pattern for all three groups. On the session preceding transfer, the 20th session of training, an analysis of variance for errors and for latencies revealed that there were no significant differences between the three groups $(\mathrm{F} /$ errors $(2,21)=0.26 ; \mathrm{F} /$ latency $(2,21)=2.50)$.

A similar analysis of variance for errors and for latencies was carried out on the 22nd session of training, the session following the transfer session. The performance of the three groups was essentially identical, and no significant differences were revealed (F/errors $(2,21)=0.22 ; F /$ latency $(2,21)=0.06)$. Consequently, the analysis of treatment effects of the three different transfer procedures has been confined to the transfer session.

\section{The Transfer Session}

Determining the Parameters for Analysis. Stimulus control was exhibited on the S2 dimension prior to the termination of the transfer session by $\mathrm{Ss}$ in all three groups. The manner of acquisition of stimulus control was quite different between groups. In the stimulus-delay group, the number of trials to the compound stimulus preceding transfer to the S2 dimension was not predetermined. These Ss determined when they transferred by shifting their response latencies such that they responded repeatedly to the $S 2$ dimension prior to the scheduled onset of the compound. As shown in Table 1, the stimulus-delay Ss transferred to the $\mathrm{S} 2$ dimension after receiving 17.29 trials to the compound stimulus, with a mean of 21.75 trials. The stimulus-compounding procedure required that the number of trials that these Ss be presented the compound stimulus be determined in advance by the $\mathrm{E}$. The stimulus-compounding Ss received the compound display for 29 trials, the maximum number of trials preceding transfer by any $S$ in the stimulus-delay group. The

Table 1

The Juxtaposition of Subjects in the Transfer Session

\begin{tabular}{|c|c|c|c|c|c|}
\hline$S$ & $\begin{array}{l}\text { Trials } \\
\text { to } S 1\end{array}$ & $\begin{array}{l}\text { Trials } \\
\text { to Com- } \\
\text { pound }\end{array}$ & $\begin{array}{l}\text { Number } \\
\text { of Com- } \\
\text { pound } \\
\text { Trials* }\end{array}$ & $\begin{array}{c}\text { S2 Trials } \\
\text { in Analysis }\end{array}$ & $\begin{array}{c}\text { Remain- } \\
\text { ing S2 } \\
\text { Trials }\end{array}$ \\
\hline \multicolumn{6}{|c|}{ Stimulus Delay } \\
\hline 1 & $1-10$ & $11-27$ & 17 & $28-48$ & $49-60$ \\
\hline 2 & $1-10$ & $11-29$ & 19 & $30-50$ & $51-60$ \\
\hline 3 & $1-10$ & $11-29$ & 19 & $30-50$ & $51-60$ \\
\hline 4 & $1-10$ & $11-30$ & 20 & $31-51$ & $52-60$ \\
\hline 5 & $1-10$ & $11-31$ & 21 & $32-52$ & $53-60$ \\
\hline 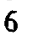 & $1-10$ & $11-31$ & 21 & $32-52$ & $53-60$ \\
\hline 7 & $1-10$ & $11-38$ & 28 & $39-59$ & 60 \\
\hline 8 & $1-10$ & $11-39$ & 29 & $40-60$ & - \\
\hline \multicolumn{6}{|c|}{ Stimulus Compounding: All Subjects } \\
\hline & $1-10$ & 11.39 & 29 & $40-60$ & - \\
\hline \multicolumn{6}{|c|}{ Abrupt Transfer: All Subjects } \\
\hline & $1-10$ & None & None & $11-30$ & $32-60$ \\
\hline
\end{tabular}

$*_{\Sigma}=174$, mean $=21.75$ 
Table 2

The Distribution of Errors During the Transfer Session

\begin{tabular}{lccc}
\hline & $\begin{array}{c}\text { Stimulus } \\
\text { Delay }\end{array}$ & $\begin{array}{c}\text { Stimulus } \\
\text { Compounding }\end{array}$ & $\begin{array}{c}\text { Abrupt } \\
\text { Transfer }\end{array}$ \\
\hline S2 Dimension & 0 & 0 & 0 \\
Distribution & 0 & 2 & 2 \\
of Errors & 0 & 3 & 3 \\
& 0 & 3 & 6 \\
\multicolumn{1}{c}{ Total } & 0 & 5 & 9 \\
\multicolumn{1}{c}{ Mean } & 1 & 7 & 11 \\
& 1 & 7 & 15 \\
Compound Display & 4 & 8 & 18 \\
Distribution & 0.750 & 35 & 64 \\
of Errors & 1 & 4.375 & 8.000 \\
& 2 & 0 & \\
& 2 & 0 & \\
& 3 & 2 & \\
& 3 & 3 & \\
& 4 & 3 & \\
\multicolumn{1}{c}{ Total } & 4 & 4 & \\
Mean & 3 & 5 & \\
\hline
\end{tabular}

Note-Statistical probability: $F(2,21)=6.12, p<.01$

stimulus-compounding $\mathrm{Ss}$ then were presented the $\mathrm{S} 2$ dimension on the 21 trials remaining in the session. The stimulus-compounding Ss only received 21 trials to the S2 dimension, thus the analysis of performance on the S2 dimension was restricted to the intial 21 trials for the Ss in the other two groups as indicated in Table 1.

An Analysis of Errors to the Compound. The Ss in both the stimulus-delay and the stimulus-compounding groups were trained on a compound stimulus. The stimulus-delay Ss received a mean of 21.75 trials to the compound, and the stimulus-compounding Ss all received 29 trials to the compound. The Ss in both groups made errors to the compound stimulus. As may be seen in Table 2, Ss in the stimulus-delay group made from one to five errors to the compound with a mean of 3.00 errors. The stimulus-compounding Ss made from zero to seven errors to the compound with a mean of 3.00 errors. While the errors exhibited by the stimulus-delay Ss were concentrated over a fewer number of trials, nearly all the errors for both groups occurred on the initial trials to the compound. Essentially, there was no difference in the distribution of errors to the compound engendered by each procedure. The data suggest that, in both groups, the presentation of a novel stimulus (e.g., the S2 dimension of the compound display) disrupted stimulus control and the magnitude of this disruption was equivalent for both procedures.

An Analysis of Errors to the S2 Dimension. Figure 2 Presents the mean number of errors to the $\mathrm{S} 2$ dimension for the Ss in each group. The mean value of 0.75 errors for the stimulus-delay group demonstrates that Ss receiving Touchette's procedure had virtually acquired the $\mathrm{S} 2$ discrimination while responding to the compound display. As can be seen in Table 2, of the eight $S$ s in this group, five made no errors to the $\mathrm{S} 2$ dimension.

The stimulus-compounding Ss averaged 4.375 errors to the $\mathrm{S} 2$ dimension. Only one $\mathrm{S}$ in this group was errorless, as indicated in Table 2 . Thus, the progressive delay of the compound stimulus, as experienced by the stimulus-delay Ss, was successful not only in indicating the point by which the S2 dimension had acquired stimulus control, but also in facilitating the acquisition of the $\mathrm{S} 2$ discrimination.

The abrupt-transfer Ss averaged 8.000 errors to the S2 dimension. This suggests that the 29 trials to the compound stimulus, as experienced by the stimulus-compounding group, also facilitated learning the $\mathbf{S} 2$ discrimination.

As presented in Table 2, the distribution of errors to the S2 dimension was significantly different between the three groups $[\mathrm{F}(2.21)=6.12, \mathrm{p}<.01]$.

An Analysis of Latency to the S2 Dimension. The development of stimulus control during transfer may also be expressed by latency. Barrera and Eckerman (1972), Jenkins (1961), Terrace (1963a, b), Moody, Stebbins and Iglover (1971), and Stebbins and Reynolds (1964) have previously employed the latency of response as an indication of stimulus control. During the acquisition of a discrimination, response latency increases to the stimulus followed by extinction and

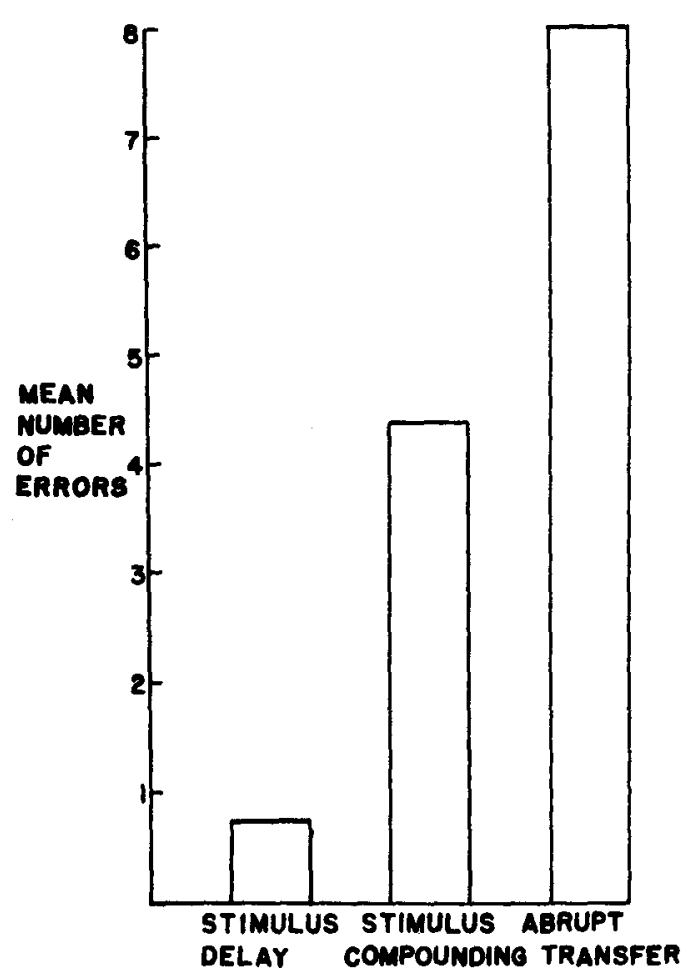

Fig. 2. Mean number of errors for $\mathrm{Ss}$ in each group on the 21 trials to the $\mathbf{S 2}$ dimension. 


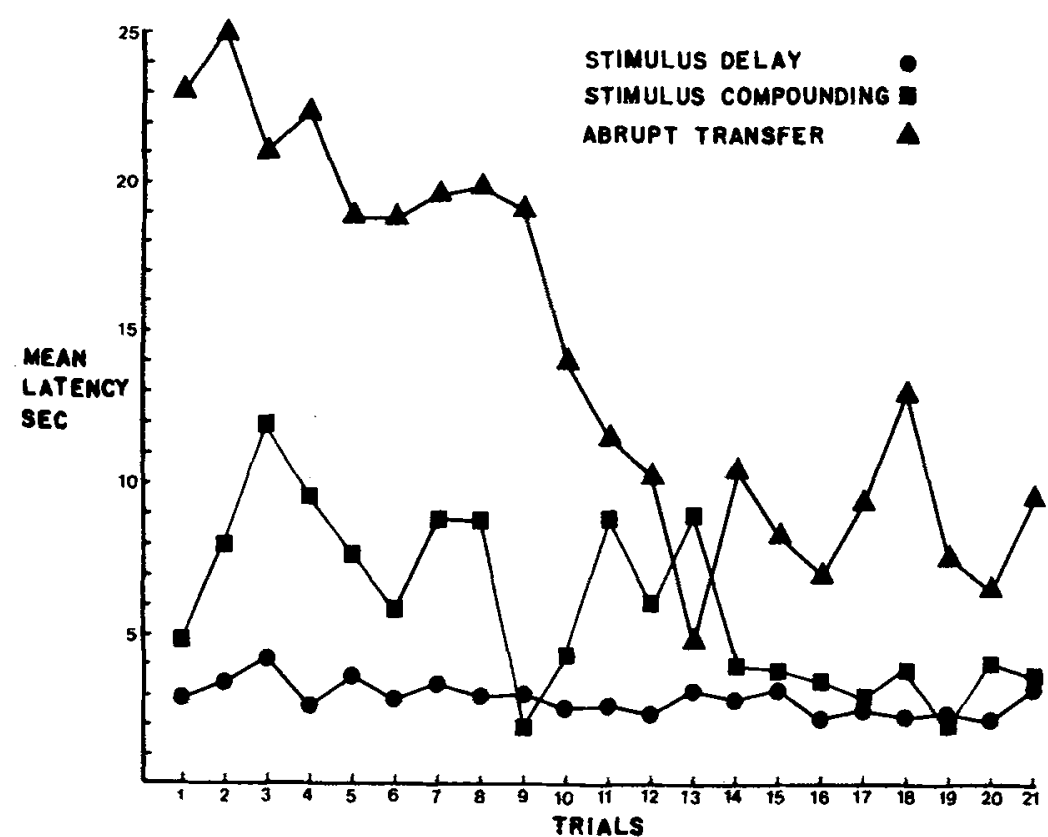

Fig. 3. Mean response tendency for Ss in each group on the 21 trials to the S2 dimension. decreases to asymptotic stable values to the stimulus correlated with positive reinforcement. Figure 3 presents the mean latency for the 21 trials on the $\mathrm{S} 2$ dimension for the Ss in each group.

The stimulus-delay Ss had the most homogeneous distribution of latencies. Ss in this group exhibited near asymptotic latencies to the S2 dimension immediately following transfer. The mean latency for this group was $2.90 \mathrm{sec}$. The observed distribution of latencies reaffirms the analysis of errors, demonstrating that the stimulus-delay group had essentially acquired the S2 discrimination while responding to the compound stimulus.

The stimulus-compounding Ss displayed intermediate latencies which gradually decreased to asymptote by the 14th trial. The mean latency for this group was $5.87 \mathrm{sec}$. The longer latencies observed for the initial 13 trials reflect that the stimulus-compounding Ss learned less about the $\mathrm{S} 2$ dimension embedded in the compound than did the $S s$ in the stimulus-delay group.

As presented in Fig. 3, the latencies for the abrupt-transfer group decreased over trials from $25 \mathrm{sec}$ to the 8 -sec range. The mean response latency was $14.3 \mathrm{sec}$. The very long latencies at the onset of the session again indicate the absence of stimulus control produced by the abrupt-transfer procedure.

The group latency curves, as presented in Fig. 3, are representative of the performance of the $S s$ in each treatment. None of the $S s$ in the abrupt-transfer procedure displayed the near asymptotic latencies displayed by the stimulus-delay group. Two of the stimulus-compounding Ss displayed latencies within the range exhibited by the stimulus-delay group. The two Ss displayed the fewest number of errors to the $S 2$ discrimination exhibited by the stimulus-compounding group. The data support the analysis that latency to response reflects the stimulus control acquired by the $S 2$ discrimination during training to the compound stimulus.

The violation of homoscedasticity in the latency data called for nonparametric statistical treatment. A Kruskal-Wallis analysis of variance demonstrated a significant difference in latency due to the main effect of groups $\left(\chi^{2}(2)=17.12, p<.001\right)$. A corresponding analysis by trials demonstrated that the abrupt-transfer and the stimulus-compounding procedures generated significantly longer latencies to the initial trials to the S2 dimension compared to subsequent trials $\left(\chi^{2} /\right.$ abrupt (2) $=44.55, \mathrm{p}<.001 ; \chi^{2} /$ compound $\left.(2)=35.62, \mathrm{p}<.02\right)$. The latencies for the stimulus-delay procedure did not change as a function of trials $\left(\chi^{2} /\right.$ delay $\left.(2)=12.44\right)$. This statistically verifies the relationship displayed in Fig. 3.

\section{DISCUSSION}

The results clearly indicate the superiority of Touchette's progressive-delay procedure. Specifically, the stimulus-delay technique not only successfully indicated the trial by which the second discrimination had acquired stimulus control, but it also facilitated acquisition. However, the facilitation expressed by the stimulus-delay procedure lay not in its capacity to introduce a stimulus dimension without disrupting stimulus control, but to reduce the probability of an error to the second discrimination subsequent to transfer.

The occurrence of errors to the compound exhibited by the stimulus-delay Ss contrasted with the performance of Touchette's Ss, who maintained control to the compound. This discrepancy may well be accounted for by the experimental history of 
Touchette's Ss. Not only had each S participated in prior research but, typically, these children experience similar discriminative stimuli within their research ward. Touchette did not elaborate on the extent of the children's experience, but it is reasonable to assume that the wavelength dimension had become an overlearned generalized discrimination. This assumption is supported by the robustness of Touchette's red-white discrimination. Touchette's $\mathrm{S}$ errorlessly executed a series of reversals on the $\mathrm{S} 2$ dimension. In this procedure, the reversal began by superimposing the most recently acquired S+ on the white background. In every case, the S "ignored" the most recently learned stimuli and responded correctly according to the original discrimination. This suggests that Touchet te's undefined pretraining had established a hierarchical relationship between his discriminations with his original dimension superior. Schusterman (1967) employed pretraining to establish a size discrimination as dominant over a form discrimination in sea lions. Following pretraining, Schusterman, like Touchette, was able to obtain a series of errorless transfers, reversing the stimuli on the form dimension.

The stimulus-compounding treatment parallels the blocking paradigm in the aversive-control literature. Johnson and Cumming (1968), in adopting the blocking paradigm to the appetitive situation, pretrained pigeons on either a red/green wavelength discrimination, or on a vertical/horizontal line-tilt discrimination. Ss were subsequently trained on the compound stimulus display. A generalization test in extinction revealed that $80 \%$ of the responses occurred to the stimulus which was positive during pretraining. Thus, pretraining on a single-stimulus dimension "blocked" learning about the novel stimulus dimension. While blocking is certainly a robust phenomenon (Kamin, 1969), it appears to be an overstatement to suggest that nothing is learned about the novel dimension. Stimulus-compounding Ss acquired the $\mathbf{S} 2$ discrimination, making only half as many errors as the $\mathrm{Ss}$ in the abrupt-transfer group. In a successive discrimination, Terrace (1963b) has reported a similar facilitation for the compound procedure.

While the stimulus-compounding Ss did acquire the S2 discrimination, the latency and error data suggest that the synchronous presentation of the elements of the compound display is not an optimal procedure. In the stimulus-compounding group, the $\mathrm{S} 2$ dimension was presented in strict temporal contiguity with the $\mathrm{S} 1$ dimension. The S2 stimuli were redundant in that they yielded no prediction about the availability of reinforcement not already provided by the $S 1$ dimension. In the stimulus-delay group, this redundancy was removed by the progressive increase in the interval from trial onset to the presentation of $\mathrm{S} 1$. The analysis suggests that this feature functions in the following manner: First, when the trial is initiated, the keys light up and the $S$ orients toward the keys. This orientation confronts the $S$ with a novel discrimination which becomes solvable when the $\mathrm{S}$ attends to the $\mathrm{S} 1$ elements of the compound. The increase in error and latency demonstrate that the Ss in both groups did attend to the S2 dimension when first presented. However, the progressive-delay feature maximized the probability that the stimulus-delay Ss would continuously attend to the S2 dimension across trials. Attending to the positive stimulus of the S2 dimension is secondarily reinforced by the presentation of the S+ of the Sl dimension, and primarily reinforced by the subsequent delivery of food. As this association is formed, the $\mathrm{S} 2$ dimension acquires a predictive value. If the $S$ has learned that the vertical line appears on a red background, it may respond faster to the wavelength dimension by positioning itself correctly according to the wavelength dimension.

Unfortunately, not all the Ss in the stimulus-delay group were systematically observed during transfer. However, casual observation substantiated the above analysis. Prior to transfer, the Ss positioned themselves correctly in front of the S+ key, as cued by the S2 dimension, and all the Ss observed made aborted keypecks to the $\mathrm{S} 2$ dimension while waiting for the $\mathrm{S} 1$ presentation. Touchette (1971) reported the equivalent observation. His Ss placed their hands on the S+ panel in "anticipation" of the S1 presentation.

This research demonstrates that Touchette's progressive-delay technique may successvully indicate the trial by which infrahuman Ss have acquired a novel discrimination. This feature of Touchette's procedure is of considerable interest in that it allows investigators to improve instructional techniques by minimizing the redundancy in stimulus progressions. The number of trials to the compound might also serve as an index of the difficulty of various discriminations. Prior to the present research, however, it was unclear as to whether Touchette's technique was primarily a measuring device or if it assisted in the acquisition of the stimulus control it was measuring. The data suggest that the removal of the redundancy between the original and the novel stimulus that served as the mechanism to identify the point of transfer also served to facilitate the acquisition of the novel dimension. However, the occurrence of errors to the compound appears to be a function of the organisms' pretraining and not of the stimulus-delay procedure.

\section{REFERENCES}

Barrera, F. J., \& Eckerman, D. A. Response latencies in a complex discrimination. Paper presented at the Eastern Psychological Association Convention, Boston, A pril 1972.

Gellerman, L. W. Chance orders of alternating stimuli in visual discrimination experiments. Journal of Genetic Psychology, 1933. 42, 207-208.

Jenkins, $H$. M. The effect of discrimination training on extinction. Journal of Experimental Psychology, 1961, 61. 111-121

Johnson, D., \& Cumming, W. Some determiners of attention. Journal of the Experimental Analysis of Behavior, 1968, 11 $157-166$.

Kamin, L. J. Selective association and conditioning. In N. J. 
Mackintosh and W. K. Honig (Eds.), Fundamental issues in associative learning, Halifax: Dalhousie University Press, 1969. Moody, D. B., Stebbins, W. C., \& Iglover, C. Auditory generalization gradients for response latency in the monkey. Journal of the Experimental Analysis of Behavior, 1971, 16, 105-111.

Moore, R., \& Goldiamond, I. Errorless establishment of a visual discrimination using fading procedures, Journal of the Experimental A nalysis of Behavior, 1964, 7, 269-272

Ray, B. A. The course of acquisition of a line tile discrimination by rhesus monkeys. Journal of the Experimentai A nalysis of Behavior, 1967, 10,17-33.

Reynolds, G. S. Attention in the pigeon. Journal of the Experimental A nalysis of Behavior, 1961,4, 203-208.

Schusterman, R. Serial discrimination learning with and without errors by the California sea lion. Journal of the Experimental Analysis of Behavior, $1966,9,595-600$.

Schusterman, $R$. Attention shift and errorless reversal learning by the California sea lion. Science, $1967,156,833-835$.

Stebbins, W. C., \& Reynolds, R. W. Note on changes in response latency following discrimination training in the monkey. Journal of the Experimental Analysis of Behavior, 1964, 7, 229-231.

Stoddard, L. T. An observation on stimulus control in a tilt discrimination by children. Journal of the Experimental
Analysis of Behavior, 1968, 11, 321-324.

Terrace, H. Discrimination learning with and without "errors", Journal of the Experimental Analysis of Behavior, 1963a, 6 , 1-27.

Terrace, H. Errorless transfer of a discrimination across two continua. Journal of the Experimental Analysis of Behavior $1963 \mathrm{~b}, 6,223-232$

Touchette, $P$. The effects of graduated stimulus change on the acquisition of a simple discrimination in severely retarded boys. Journal of the Experimental A nalysis of Behavior, 1968 . $11,39.48$

Touchette, P. Tilted lines as complex stimuli. Journal of the Experimental A nalysis of Behavior, 1969, 12, 211-214.

Touchette, P. Transfer of stimulus control: Measuring the moment of transfer. Journal of the Experimental Analysis of Behavior, 1971, 15, 347-354

Westbrook, R., \& Miles, C. The effect of a fading procedure upon the acquisition of control by an overshadowed auditory feature. Journal of the Experimental Analysis of Behavior $1970,13,179-185$

(R eceived for publication October 3, 1973; revision accepted August 6,1974 .) 